\title{
Transport Properties of the Diluted Lorentz Slab
}

\author{
Hernán Larralde *, François Leyvraz *,

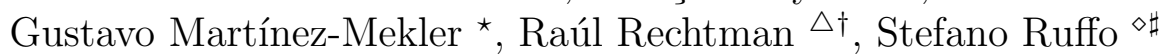 \\ $\star$ Centro de Ciencias Fisicas, UNAM, Apdo. Postal 48-3, \\ 62251 Cuernavaca, Morelos, Mexico \\ $\triangle$ Centro de Investigación en Energía, UNAM \\ 62580 Temixco, Morelos, Mexico \\ $\diamond$ Dipartimento di Energetica "S. Stecco", Università di Firenze, via S. Marta 3, \\ 50139 Firenze, Italy, INFM and INFN
}

\begin{abstract}
We study the behavior of a point particle incident from the left on a slab of a randomly diluted triangular array of circular scatterers. Various scattering properties, such as the reflection and transmission probabilities and the scattering time are studied as a function of thickness and dilution. We show that a diffusion model satisfactorily describes the mentioned scattering properties. We also show how some of these quantities can be evaluated exactly and their agreement with numerical experiments. Our results exhibit the dependence of these scattering data on the mean free path. This dependence again shows excellent agreement with the predictions of a Brownian motion model.
\end{abstract}

PACS number(s): 05.40.+j, 05.45.+b, 05.60.+w

\section{INTRODUCTION}

The Lorentz gas [1] is one of the fundamental kinetic theory models [2], and many of its ergodic properties are well known [3]. In a previous paper [4] we investigated the scattering and transmission properties of an array of disks centered on a finite triangular lattice, a Lorentz slab. There, we studied the validity of approximating the motion of such a particle by a diffusive process [5]. Specifically, we considered samples of finite thickness $L$ along the $x$ axis, but infinite along the $y$ axis. We then looked at the behavior of a particle incident on the sample from the left and measured the probability $T$ of being transmitted to the right, the reflection probability $R$ and the average residence time $\langle\tau\rangle$. We found a very satisfactory agreement with the predictions of one dimensional Brownian motion for all these quantities when the distance between scatterers is small enough to prevent any particle from moving in an arbitrarily long straight line (the so-called finite horizon case). When such unbounded motion is possible we found that normal diffusive behavior did not arise. Rather, a complex pattern of logarithmic corrections was found for the various quantities of interest. A modification of the diffusive model considering Lévy walks as suggested in Refs. [6,7] explains such features. 
Here we study the situation in which the system is randomly diluted, but the scatterers are still placed on the the sites of a triangular lattice. That is, we consider the case in which a fraction $f$ of the cells of the periodic array is occupied by scatterers and the rest is empty. In such a system, the particle can always take steps of arbitrary length, but the horizon is said to be finite if the distribution of free paths has an exponential cutoff. This happens when the corresponding system with $f=1$ has finite horizon, i.e., the only large steps that occur in the diluted system are related to the absence of a large number of scatterers, which is an exponentially improbable event. In this paper, we restrict ourselves to this finite horizon case.

The importance of looking at the randomly diluted case, is that it provides a controlled way of varying the mean free path - and hence the diffusion constant - over an arbitrarily large range, without affecting the property of having a finite horizon and hence a normal diffusive behavior. This therefore allows us to check the correspondence with diffusion models much more thoroughly. Further, it shows that the correspondence between classical deterministic motion and Brownian motion, as proved rigorously in Ref. [5] for the case of periodic billiards, does not, in fact, require strict periodicity.

This paper is organized as follows: In Sec. II we describe in detail how the numerical experiments are performed. In particular, we define two different ways in which we introduce disorder in the system, namely quenched and annealed. In Section III, we discuss the average transport properties, such as transmission probability, mean free path and average scattering time. We find that the last two quantities can be evaluated exactly using a relationship due to Kac [8]. We display the numerical results for these quantities as well as the predictions using the Kac formula and the diffusion model. For the sake of completeness, we reproduce the derivation of the Kac formula in Appendix A. In Sec. [V] we discuss the distribution functions of free paths, of residence times and of heights of exit of transmitted particles. In Appendix B we derive the latter two distributions for the diffusion model. Finally in Sec. $\square$ we present our conclusions.

\section{MODEL SETUP}

The geometric arrangement of the scatterers in the undiluted $(f=1)$ system is the following: each scatterer is a disk of unit radius, the centers of which form a triangular lattice. The slab is infinite in the vertical direction and is characterized by the number $L$ of columns and the minimal separation $w$ between the disks. Dilution is then obtained by occupying only a fraction $f$ of the sites of the array with disks of unit radius. A typical scatterer configuration is shown in Fig. 11. The left and right "sawtooth" borders are the outer sides of the hexagonal cells attached to each site of the lattice.

Particles are launched from randomly chosen positions along the left border. Each incident particle has a different impact parameter $b$, defined here as the distance between the initial position and the horizontal line passing through the center of the scatterer in the cell. The angles of incidence $\theta$ measured with respect to the side from which the particle is launched, are distributed in the interval $[0, \pi]$ in such a way as to make $\cos \theta$ uniformly distributed. This choice reproduces initially the Liouville measure in the Birkhoff coordinates. The particles move freely except for elastic collisions at the boundary of the disks. 
In the undiluted case $(f=1)$, if the separation between scatterers $w$ is small, $0<w<$ $w_{c}=(4 / \sqrt{3}-2)=0.3094 \ldots$, the length of free motion of the particles is bounded and they "see" a finite horizon. All the numerical experiments discussed in this paper are performed in this range. On the other hand, in the diluted case, arbitrarily long paths without collisions can exist also for $0<w<w_{c}$, but their contribution to the diffusion constant remains finite.

As far as the dilution process itself is concerned, we realize it in two different ways corresponding to the usual distinction between quenched and annealed disorder. The annealed disorder is obtained by choosing with probability $f$ the cell to be occupied at the moment at which the particle enters the cell. Thus, when the particle eventually revisits a given cell, its occupancy status may be different. This way of introducing disorder involves a simultaneous average over the dynamical and the disorder variables. On the other hand, we also performed dilution in the more realistic quenched case, in which a sample is first created, for which all cells are either occupied or empty with probability $f$ and averages are taken over many realizations of disorder.

\section{AVERAGE TRANSPORT PROPERTIES}

We begin this Section by showing a derivation of a formula for the mean free path $\lambda$ as a function of the geometrical parameter $w$ and dilution $f$. This quantity can be evaluated in terms of phase space integrals using Kac formula [8], which assumes ergodicity. Indeed, as shown in Appendix A, for a particle moving freely at unit velocity in a bounded domain of area $S$, the average return time $\langle\mathcal{T}\rangle$ to a boundary segment of length $P$ is

$$
\langle\mathcal{T}\rangle=\frac{\pi S}{P} .
$$

For the Lorentz gas, choosing the boundary segment as the perimeter of all disks, the average return time coincides with the mean free time between two collisions. Since the particles move with unit velocity, this also coincides with the mean free path $\lambda$. Considering a finite array of Wigner-Seitz hexagonal cells with $L$ columns and $M$ rows, the total area of the domain is $L M C$, where $C=(2+w)^{2} \sqrt{3} / 2$ is the cell area. From this we must subtract the area occupied by the unit radius disks, i.e. $\pi f L M$. The total perimeter is $P=2 \pi f L M$. Hence, from Eq. (四) and the above considerations

$$
\lambda=\frac{\sqrt{3}}{4 f}(w+2)^{2}-\frac{\pi}{2} .
$$

Note that this holds for any value of $M$ and hence extends trivially to the case we consider, where $M$ is infinite. The usual derivation of Eq. (1) only applies to the quenched case, i.e. to a scatterer configuration that is fixed in time. However, the arguments we give in Appendix A show how to extend it to the annealed case as well. Numerical experiments confirm this finding, for both quenched and annealed disorder. In Fig. 2 we display the results for quenched disorder, since the annealed data are identical.

The slab is infinite in the $y$ direction and the collisions are elastic, hence every particle that enters the slab must eventually leave it, except for a set of zero measure which goes asymptotically to bounded orbits inside the slab. Thus, in practice, a particle that enters 
the slab collides with some of the obstacles and is ultimately transmitted or reflected. From Eq. (1) one can obtain the average residence time $\langle\tau\rangle$ as a function of $w, f$ and $L$, if one now takes the border of the slab as the boundary segment. For a slab with $L$ columns and $M$ rows the perimeter is $M 4(2+w) / \sqrt{3}$ and the total area of the domain is as before. Hence

$$
\langle\tau\rangle(f, w, L)=\left(\frac{3 \pi(2+w)}{8}-\frac{\sqrt{3} \pi^{2} f}{4(2+w)}\right) L=B(f, w) L .
$$

This equation defines the quantity $B$. As above (see Eq. (2)), the result is independent of $M$. We verified numerically the linear growth of $\langle\tau\rangle$ with $L$ and found it to be valid, as expected, also for small values of $L$. From these experiments we obtained the values of $B$ shown in Fig. 3 for quenched disorder together with Eq. (3). The agreement in the annealed case (not shown) is even more satisfactory. Note that $B$ does not diverge as $f \rightarrow 0$, as opposed to the mean free path.

Let us now compare these exact results with the predictions of a model based on Brownian motion. We assume that the particles incident from the left penetrate a given distance $a$ into the sample, after which they forget everything about the way they were injected into the system and diffuse with diffusion constant $D$. Under these assumptions, the quantities of interest can be evaluated exactly in terms of $a$ and $D$ and compared with the exact results obtained above. Thus, the average time to reach either side starting at a distance $a$ from the left side is given by 10,11

$$
\langle\tau\rangle(L)=\frac{a(\ell L-a)}{2 D}
$$

where $\ell=\sqrt{3}(1+w / 2)$ is the horizontal separation between columns of the slab. One sees therefore that the form of the $L$ dependence in the diffusive case is slightly different from the exact one, Eq. (3), since in that case the proportionality to $L$ is exact over the whole range of $L$. On the other hand, in Eq. (雨), a constant term appears which is negligible in the limit $a / \ell \ll L$. Since this is the limit for which diffusion is expected to be a valid description, this is not a serious problem. On the other hand, this allows to evaluate the ratio $a / D$ exactly in terms of $f$ and $w$, via

$$
\frac{a}{D}=\frac{2 B(f, w)}{\ell}
$$

It is well known that it is not possible to obtain exact evaluations of the same sort for the diffusion constant $D$, since its value can be shown to depend in a detailed manner on the specific dynamics involved. Indeed,

$$
D=\lim _{\epsilon \rightarrow 0} \int_{0}^{\infty} \overline{\dot{x}(0) \dot{x}(t)} e^{-\epsilon t} d t
$$

where the bar denotes an average over the realizations of the disorder. From this it follows that an analytical expression for $D$ in terms of simple phase space expressions is impossible. However, an order of magnitude estimate for $D$ can be given. Since the mean square distance grows linearly with time, and since the only microscopic length scale is the mean free path, we are led to estimate $D t_{0}$ by the square of the mean free path, where $t_{0}$ is the time needed 
for the particle to cover a mean free path. In our system, the velocity of the particle is constant and equal to one, therefore the result is that $D$ is of the same order as $a$, both being of the order of the mean free path. This is indeed consistent with Eq. (5). It should be noted that the ratio of the two dynamics dependent quantities $a$ and $D$ depends only on the geometric features of the system.

Let us now turn to another average transport property, for which no exact expression is available, namely the transmission probability $T$ as a function of $L$. One finds that asymptotically, in the limit $L \gg 1$,

$$
T(f, w, L)=\frac{A(f, w)}{L}
$$

In Fig. 4 , we show that $A$ grows almost linearly with $\lambda$, for large values of $\lambda$. This can readily be understood in terms of the diffusive model. Indeed, in the case of a diffusing particle being injected at a distance $a$ from the left-hand side of the slab, the probability that it be transmitted to the right-hand side without being absorbed first at the left-hand side $T_{d}$ is well-known to be [10,1]

$$
T_{d}(L)=\frac{a}{\ell L}
$$

From this we see that $A$ can be identified with a quantity which, as argued above, is expected to scale as the mean free path. Note further that whereas $\langle\tau\rangle$ and $\lambda$ are identical in the quenched and annealed cases, this is not true for the transmission probability, which shows significant differences for the two forms of disorder.

\section{DISTRIBUTION FUNCTIONS}

We have also studied the distributions of residence times of transmitted (respectively reflected) particles, of free paths and of the heights at which transmitted (respectively reflected) particles leave the system.

Of these, only the distribution of free paths is accessible to exact theoretical treatment. Indeed, this is nothing but an equilibrium property, and can in principle be evaluated using an integral over the perimeter of the billiard with the Birkhoff measure. However, this is not really practicable, so we have not attempted it. The distribution of mean free paths is quite irregular (see Fig. 5). This irregularity arises from the presence of resonances and the fact that there are forbidden distances. Note that, as remarked in the Introduction, the distribution decays exponentially since the horizon is finite. From the above characterization of the mean free path distribution as an equilibrium property, it readily follows that they are independent of whether the average is taken as quenched or annealed.

Now let us consider the distribution of residence times, which is not susceptible of an exact evaluation. However, it can be computed in the diffusion model. It is clearly sufficient to compute the distribution of residence times for transmitted particles $p_{\text {trans }}$ upon starting at $a$, since the corresponding distribution for reflected particles $p_{r e f l}$ arises by substituting

$a$ by $\ell L-a$ in $p_{\text {trans }}$. Defining the scaled variables $\bar{\tau}=D \tau /(\ell L)^{2}$ and $\alpha=a /(\ell L)$, the final result is, as shown in Appendix B [11] 


$$
p_{\text {trans }}(\bar{\tau} ; \alpha)=2 \pi \sum_{n=1}^{\infty} n \sin \pi n(1-\alpha) e^{-\pi^{2} n^{2} \bar{\tau}}
$$

Integrating, one obtains Eq. (4) for the average time a diffusing particle takes to reach either side. We fit this to the numerical data as follows: one first considers the average time that a transmitted particle remains in the sample. This is obtainable from the experimental distribution on the one hand, but is also given by

$$
\langle\tau\rangle_{\text {trans }}=\frac{\ell^{2} L^{2}}{6 D}
$$

This allows therefore a determination of $D$ from the data, which for $f=1$ is in good agreement with published data [9]. From this and Eq. (3) together with Eq. (4), one also obtains a value of the parameter $a$. One is then in a position to plot the theoretical curve Eq. (9) together with the empirical data. This is shown in Fig. 6, for an occupation fraction $f=0.5$. The agreement is quite good. The apparent shift between the theoretical curve and the data in reflection can be traced back to an issue of normalization involving particles reflected after a very few bounces, which therefore do not show diffusive behavior. Thus, the empirical distribution has a short-time cut-off at larger times than the corresponding diffusive model.

Finally, we measure the distribution of heights of transmitted particles. This distribution can be computed in the diffusive model. The result for transmitted particles is, as shown in Appendix B,

$$
p(\eta)=\frac{\sin \pi(1-\alpha)}{2 \alpha} \frac{1}{\cosh \pi \eta-\cos \pi(1-\alpha)},
$$

where $\eta$ is the scaled height $y /(\ell L)$ and $\alpha$ is as above. In Fig. 7 we show $p(\eta)$ for transmitted particles. The agreement is excellent. We do not show the behavior for reflected particles as it is dominated by rapid reflections.

\section{CONCLUSIONS}

Summarizing, we have studied transport properties of finite size samples of Lorentz gases in a situation in which the mean free path can be varied over a large range of values without affecting the property of normal diffusion. The mean free path was varied over a considerable range (slightly more than an order of magnitude), and the agreement with the Brownian motion model was satisfactory throughout. We have also shown how the diffusion constant and the penetration depth, which were the two dynamical parameters of our model, vary with dilution, and hence with the mean free path.

\section{ACKNOWLEDGMENTS}

We thank A. Bufetov for stimulating discussions. FL acknowledges the hospitality of the Dipartimento di Energetica of the University of Florence, Italy, where part of this work 
was carried out. This work has been partially supported by INFN, INFM, University of Florence, MURST-COFIN00 grant on Chaos and Localization in Classical and Quantum Mechanics, DGAPA-UNAM under grants IN103595, IN106597, IN112200 and CONACyT under grants 32418-E and 32173-E.

\section{APPENDIX A:}

In this Appendix, we show in detail how the exact relations Eq. (2) and Eq. (3) are obtained. To this end, we first recall the derivation of a general formula due to Kac. Consider a $(2 N-1)$-dimensional energy shell in a $2 N$-dimensional Hamiltonian phase-space and select a $(2 N-2)$-dimensional Poincaré surface that intersects all (or nearly all) trajectories on the energy shell. If such a surface cannot be found, then the phase space integrals below must be restricted to that part of the phase space which can be reached from the surface.

Parametrize each point $(p, q)$ on the energy surface using the last point $(\bar{p}, \bar{q})$ on the Poincaré surface that lies on the trajectory passing through $(p, q)$. Denote by $\mathcal{T}_{E}(\bar{p}, \bar{q})$ the time necessary to reach $(p, q)$ starting from $(\bar{p}, \bar{q})$. This defines the canonical coordinate transformation

$$
d^{N} p d^{N} q=d^{N-1} \bar{p} d^{N-1} \bar{q} d \mathcal{T} d E .
$$

The constant energy $E_{0}$ volume is hence

$$
\int d^{N} p d^{N} q \delta\left[E_{0}-H(p, q)\right]=\int d^{N-1} \bar{p} d^{N-1} \bar{q} \mathcal{T}_{E_{0}}(\bar{p}, \bar{q})
$$

Denoting by $\mathcal{N}$, the total $(2 N-2)$-dimensional phase space volume of the Poincaré surface, one immediately obtains the Kac formula for the average time to return to the surface:

$$
\begin{aligned}
\left\langle\mathcal{T}_{E_{0}}\right\rangle & =\mathcal{N}^{-1} \int \delta\left[E_{0}-H(p, q)\right] d^{N} p d^{N} q, \\
\mathcal{N} & =\int_{E=E_{0}} d^{N-1} \bar{p} d^{N-1} \bar{q} .
\end{aligned}
$$

To evaluate these integrals in the case of billiards for $N=2$ (of which the Lorentz gas with quenched disorder is a particular case), we take the Hamiltonian to be $p^{2} / 2$ and $E_{0}$ to be $1 / 2$. From this follows

$$
\int d^{2} p d^{2} q \delta\left[1 / 2-p^{2} / 2\right]=2 \pi S
$$

where $S$ is the area of the billiard. As a Poincaré surface we introduce an arbitrary subset of the billiard boundary having perimeter $P$, with the usual Birkhoff coordinates as variables $\bar{p}$ and $\bar{q}$. One then finds

$$
\int_{E=E_{0}} d \bar{p} d \bar{q}=P \int_{-\pi / 2}^{\pi / 2} \cos \theta d \theta=2 P
$$

From these two equations one derives Eq. (1) 
The above remarks are clearly limited to the case of quenched disorder. The generalization to the annealed case can be made as follows: to every point $(\bar{p}, \bar{q})$ on the Poincaré surface, add a doubly infinite sequence of zeroes and ones $\left(\sigma_{k}\right)_{k=-\infty}^{\infty}$, which we denote by $\bar{\sigma}$, with all $\sigma_{k}$ independently distributed and equal to one with probability $f$. We now define the dynamics as follows: The orbit starts from $(\bar{p}, \bar{q})$ and the cell at $t=0$ is occupied or empty according to the value of $\sigma_{0}$. The orbit then proceeds until it leaves the cell. The status of the next cell is then decided according to the value of $\sigma_{1}$ and so on. Although discontinuities arise when a trajectory crosses a vertex of the fundamental cell, the dynamics is still given by a canonical map. Thus, for a fixed sequence $\bar{\sigma}$ the formula Eq. (A3) applies. However, for a fixed sequence, we cannot easily compute the phase space volumes involved. Since we are only interested in the average of $\langle\mathcal{T}\rangle(\bar{\sigma})$ over all values of $\bar{\sigma}$, we note the following: the denominator in Eq. (A3) is independent of $\bar{\sigma}$, so that it is sufficient to average over the numerator. This average can be performed separately for each cell, and the result therefore follows trivially.

\section{APPENDIX B:}

In this Appendix, we derive Eq. (9) and Eq. (11). To this end, we first derive an analogue of Eq. (9) for the probability that a particle first exits the slab on the right side at a height $y$ and at time $t$. In order to simplify the notation, we first go over to scaled variables $\xi=x /(\ell L), \eta=y /(\ell L), \alpha=a /(\ell L)$ as well as $\bar{\tau}=D t /(\ell L)^{2}$. All distribution functions are further rescaled in such a way as to remain normalized. In these variables, this probability is given by the following expression:

$$
p_{\text {trans }}(\eta ; \bar{\tau})=-\left.\frac{\partial P_{0}(\xi . \eta ; \bar{\tau})}{\partial \xi}\right|_{\xi=1},
$$

where $P_{0}(\xi, \eta)$ is the solution of the following problem:

$$
\begin{aligned}
\frac{\partial P_{0}(\xi, \eta ; \bar{\tau})}{\partial \bar{\tau}} & =\Delta P_{0}(\xi, \eta), \\
P_{0}(\xi, \eta ; 0) & =\delta(\xi-\alpha) \delta(\eta) \quad P_{0}(0, \eta ; \bar{\tau})=P_{0}(1, \eta ; \bar{\tau})=0 .
\end{aligned}
$$

This is now solved by developing the delta function in eigenmodes of the Laplacian satisfying the boundary conditions in Eq. (B2), that is

$$
P_{0}(\xi, \eta ; \bar{\tau})=2 \sum_{n=1}^{\infty} \sin n \pi \xi \sin n \pi \alpha \int_{-\infty}^{\infty} \frac{d k}{2 \pi} e^{i k \eta} \exp \left[-\left(\pi^{2} n^{2}+k^{2}\right) \bar{\tau}\right] .
$$

The resulting expression for $p_{\text {trans }}(\eta, \bar{\tau})$ can now be integrated either over $\eta$ or over $\bar{\tau}$ to yield Eq. (9) or Eq. (11) respectively. In either case, obtaining the results in the text is now a matter of straightforward algebra. 


\section{REFERENCES}

$\dagger \quad$ e-mail: rrs@teotleco.cie.unam.mx

\# e-mail: ruffo@avanzi.de.unifi.it

[1] H.A. Lorentz, Proc. Amst. Acad. 7, 438, 585, 604 (1905).

[2] G. Gallavotti, Phys. Rev. 185, 308 (1969); E.H. Hauge, in Lecture Notes in Physics, 31 pp. 337-367 (1974).

[3] Sh. Goldstein, J. Lebowitz amd M. Aizenman, in Lecture Notes in Physics, 38 pp. $112-$ 143 (1975); G. Gallavotti, in Lecture Notes in Physics, 38, pp. 236-295 (1975); Ya. G. Sinai, Funkts. Anal. Ego Prilozh., 13, 46 (1979).

[4] H. Larralde, F. Leyvraz, G. Martínez-Mekler, R. Rechtman, S. Ruffo, Phys. Rev. E, 58 4254 (1998).

[5] L. A. Bunimovich and Ya. G. Sinai, Commun. Math. Phys. 78, 479 (1981).

[6] J.-P. Bouchaud and A. Georges, Phys. Rep., 195, 127 (1990).

[7] P. M. Bleher, J. Stat. Phys., 66, 315 (1992).

[8] M. Kac, Bull. Amer. Math. Soc., 53, 1002 (1947).

[9] C. Dellago and R. Klages, J. Stat. Phys, 101, 145 (2000).

[10] W. Feller, An Introduction to Probability Theory and its Applications, vol. 2, second edition (John Wiley, N.Y.) 1971.

[11] G.H. Weiss, Aspects and Applications of the Random Walk (North-Holland, Amsterdam) 1994. 


\section{FIGURES}

FIG. 1. Slab of diluted scatterers in a triangular array with $L=10, w=0.2$, and $f=0.7$. Point particles enter the slab on the left side saw tooth border.

FIG. 2. Mean free path shifted by $\pi / 2$ as a function of the dilution $f$ for quenched disorder. The curves are the r.h.s. of Eq. (2) again shifted by $\pi / 2$. Each point represents the average over $10^{8}$ collisions.

FIG. 3. Dependence of $B$ on $f$ for quenched disorder. The curves are Eq (3). The values of $B$ were found by adjusting Eq. (3) to the experimental data obtained by letting $10^{7}$ particles travel through slabs of lengths going from 100 to 1500.

FIG. 4. Dependence of $A$ on the mean free path $\lambda$ for $w=0.2$. Both quenched and annealed disorder are shown. The values of $A$ were found by adjusting Eq. (7) to the experimental data obtained by letting $10^{7}$ particles travel through slabs of lengths going from 100 to 1500 .

FIG. 5. Free path distribution for $w=0.2, f=0.5$ for both quenched and annealed disorder. The curves are practically superimposed. The distributions were found from $10^{8}$ collisions in each case.

FIG. 6. Distribution of residence times for reflected (a) and transmitted (b) particles for quenched disorder, $w=0.2$ and $f=0.5$. The continuous curve represents the fit described in the text. The experimental distributions were found by letting $10^{7}$ particles travel through a slab with 100 columns.

FIG. 7. Height distribution of transmitted particles for quenched disorder, $w=0.2$ and $f=0.2$. The continuous curve is the plot of Eq. (11). The experimental distribution was found by letting $10^{7}$ particles travel through a slab with 100 columns. 


\section{FIGURES}

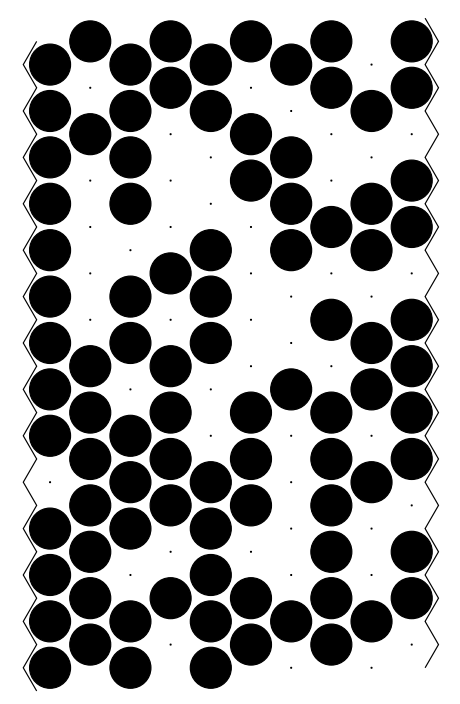

FIG. 1.

H. Larralde et al, Transport Propertiesof the Diluted Lorentz Slab 


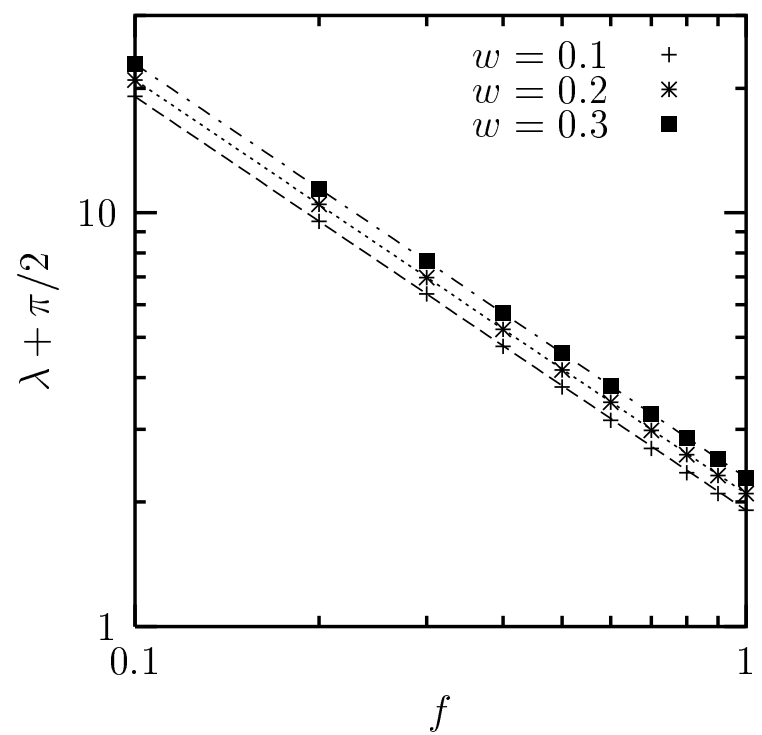

FIG. 2.

H. Larralde et al, Transport Propertiesof the Diluted Lorentz Slab 


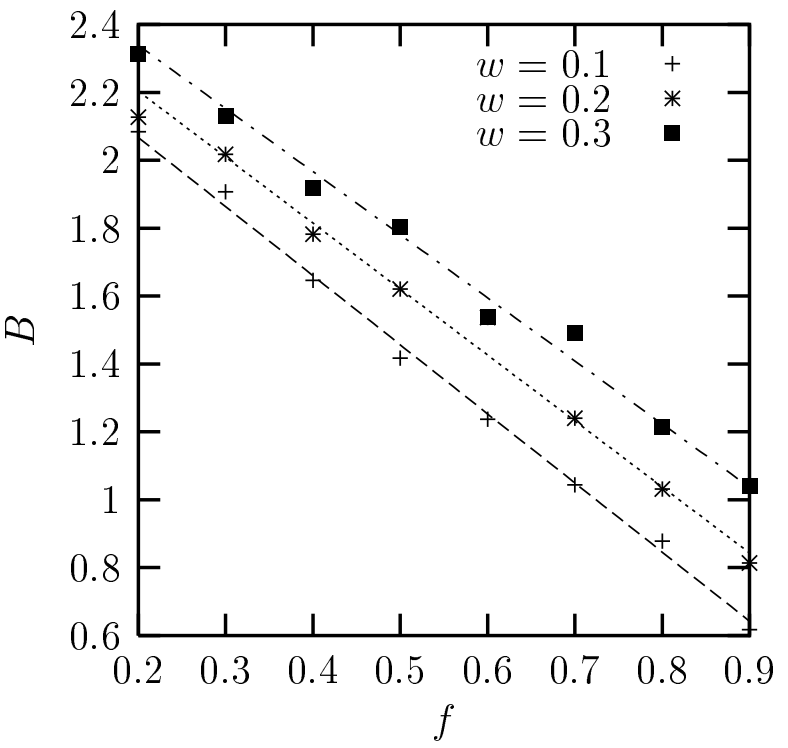

FIG. 3 .

H. Larralde et al, Transport Propertiesof the Diluted Lorentz Slab 


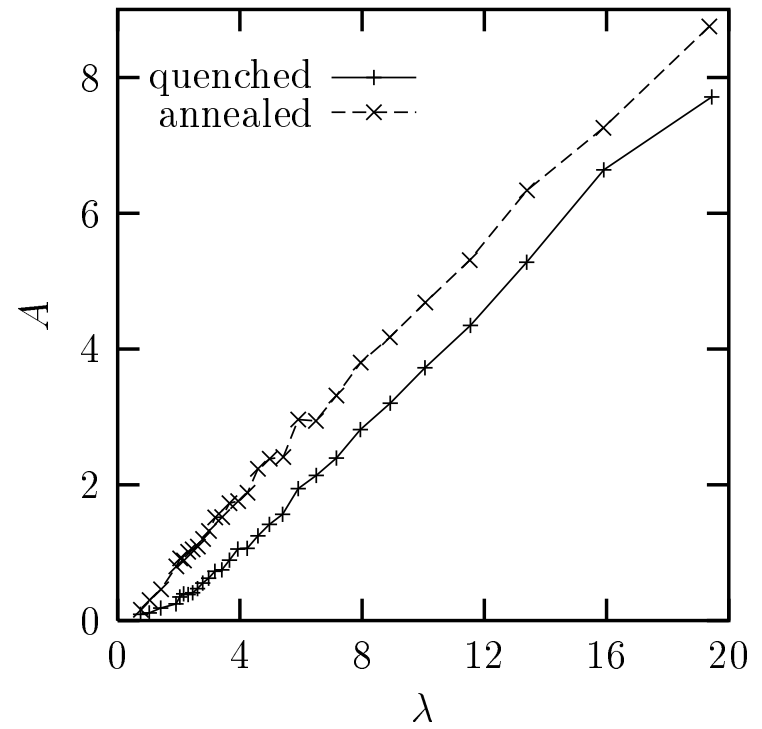

FIG. 4.

H. Larralde et al, Transport Propertiesof the Diluted Lorentz Slab 


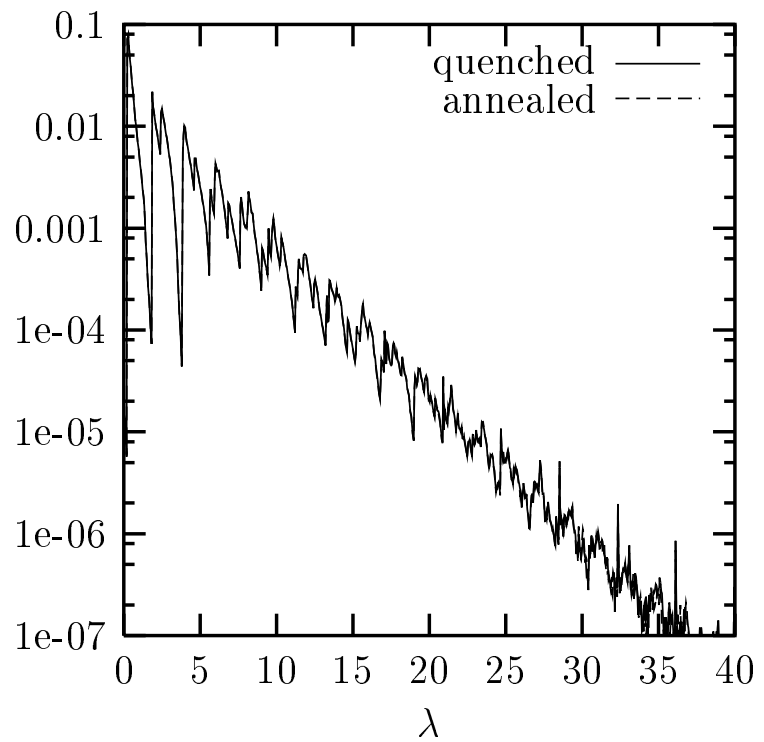

FIG. 5.

H. Larralde et al, Transport Propertiesof the Diluted Lorentz Slab 

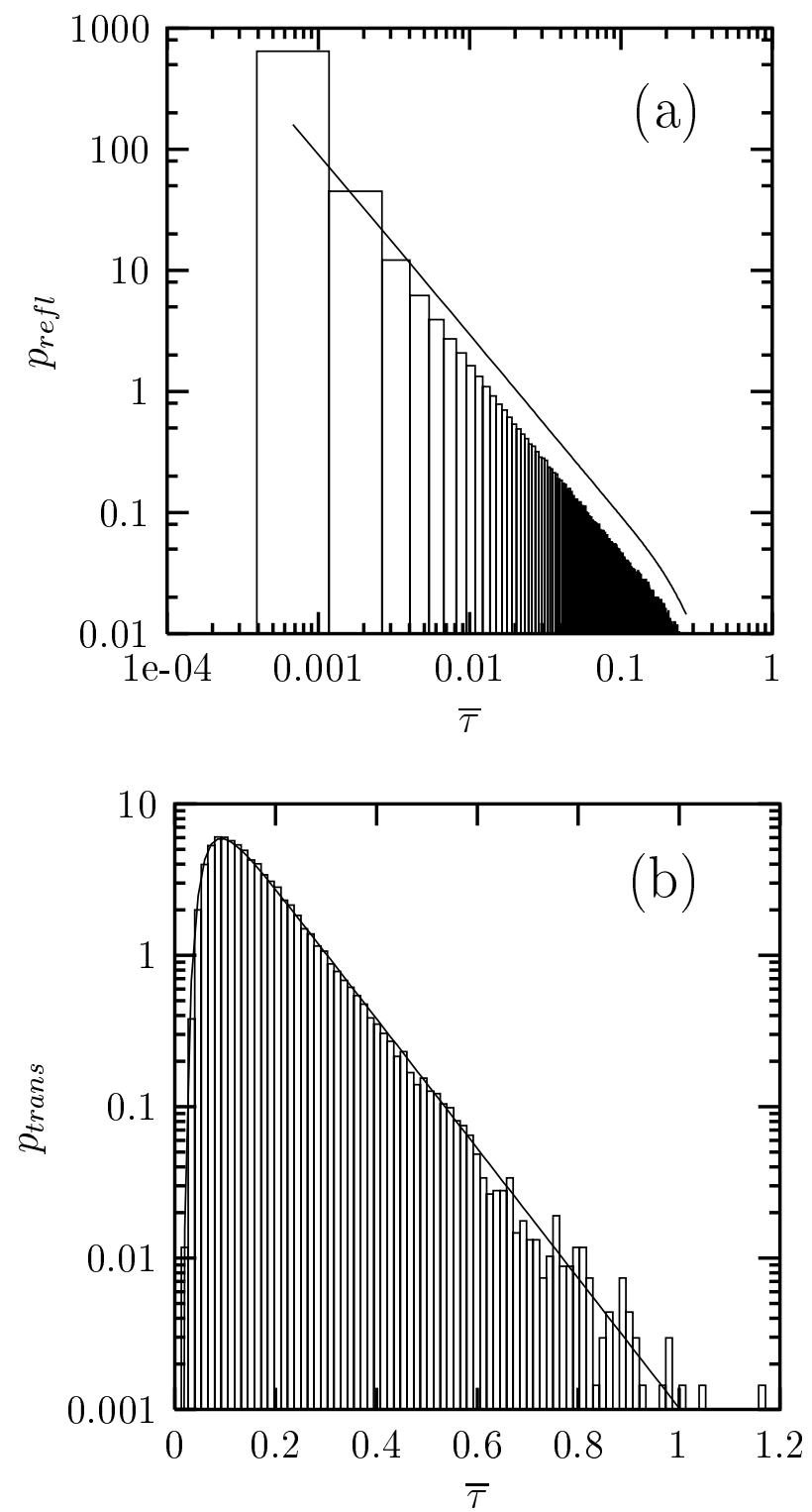

FIG. 6 .

H. Larralde et al, Transport Propertiesof the Diluted Lorentz Slab 


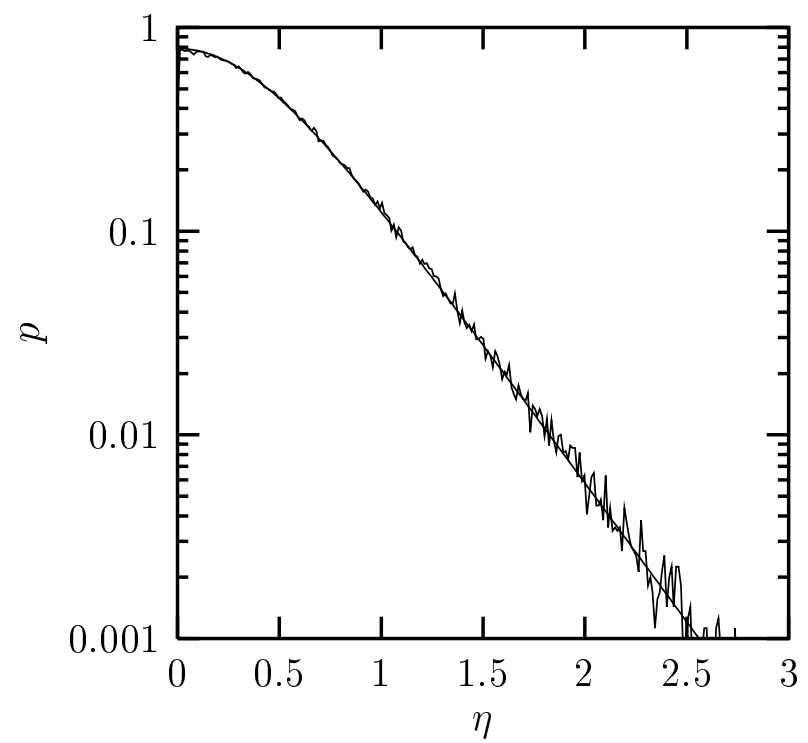

FIG. 7.

H. Larralde et al, Transport Propertiesof the Diluted Lorentz Slab 